\title{
RÖSCH, Eva Sibylle, RÖSCH, Gerhard, Kaiser Friedrich II. und sein Königreich Sizilien
}

\section{Michel Parisse}

\section{OpenEdition}

\section{Journals}

Édition électronique

URL : http://journals.openedition.org/ifha/1510

DOI : 10.4000/ifha.1510

ISSN : 2198-8943

\section{Éditeur}

IFRA - Institut franco-allemand (sciences historiques et sociales)

\section{Référence électronique}

Michel Parisse, «RÖSCH, Eva Sibylle, RÖSCH, Gerhard, Kaiser Friedrich II. und sein Königreich Sizilien », Revue de l'IFHA [En ligne], Date de recension, mis en ligne le 01 janvier 1997, consulté le 22 septembre 2020. URL : http://journals.openedition.org/ifha/1510 ; DOI : https://doi.org/10.4000/ifha.1510

Ce document a été généré automatiquement le 22 septembre 2020.

(CIFHA 


\title{
RÖSCH, Eva Sibylle, RÖSCH, Gerhard, Kaiser Friedrich II. und sein Königreich Sizilien
}

\author{
Michel Parisse
}

1 L'histoire de l'Empire à l'époque de Frédéric II continuera longtemps encore de passionner les historiens allemands, qui adjoignent au gouvernement et aux aventures du fascinant petit-fils de Frédéric Barberousse le court règne de Henri IV, dont le mariage avec Constance de Sicile a permis la fabuleuse carrière de leur fils. Voici deux ouvrages qui nous mettent en contact avec les hommes et leur période. Et d'abord ce cadeau inestimable de la maison d'édition de Sigmaringen qui met à notre disposition pour un prix tout à fait abordable une oeuvre unique, l'histoire en vers et en image que composa Pierre d'Eboli »en l'honneur de l'empereur« ou, si l'on veut, pour présenter la Sicile. Pour Th.K., que l'on connaît comme l'éditeur de diplômes siciliens, le critique des chartes de Saint-Maximin de Trèves et le collaborateur de C. BRÜHL pour une nouvelle édition des diplômes mérovingiens, et qui est ici le maître d'oeuvre de l'édition, et son présentateur, l'oeuvre de Pierre d'Eboli est à rapprocher de la Tapisserie de Bayeux comme suite d'images. Le manuscrit de 147 folios, conservé aujourd'hui à la Burgerbibliothek (on ne commettra pas l'erreur d'écrire »Bürger« au lieu de "Burger«, dont le sens à Berne est bien précis) de Berne, présente sur ses pages de gauche, au verso donc, un texte en vers, auquel correspond sur la page de droite le récit en images, coloré, mais avec une dominante verte. Pour l'édition, une page a été intercalée entre les folios, ce qui a permis d'une part, d'imprimer en face du récit en vers la transcription de ceux-ci sur une colonne et leur traduction en allemand sur une deuxième colonne, d'autre part de disposer en face des images un abondant commentaire de l'iconographie. Cette disposition rend particulièrement agréable la lecture du livre. L'histoire commence avec la mention des trois mariages du duc Roger II (1095-1154) et la naissance de Constance, fille de Roger et de Béatrice de Rethel, suivie du mariage de la même Constance avec le fils et héritier de Frédéric Barberousse, le 27 janvier 1186. Dès lors les épisodes se succèdent, mettant en scène la mort du roi Guillaume de Sicile, l'intervention de Tancrède, jusqu'au couronnement impérial de 
Henri VI (1191). On verra encore la naissance de Frédéric II, le 29 décembre 1194. Les dernières images sont toujours à l'honneur de Henri VI. Les dessins suscitent un constant ravissement, par les personnages et leurs attitudes, le cadre où ils se meuvent, leur habillement, leur comportement. Pourquoi ne pas en retenir, à titre d'exemple, cette page qui évoque l'activité de la chancellerie normande, dont on nous montre qu'on y était en mesure d'écrire des documents en grec, en sarrasin, en latin (on voit trois images avec des notaires pour chaque langue; on sait que le latin l'emportait largement); l'image du coursier (cursor) s'ajoute aux précédentes jusqu'à la remise des lettres à leur destinataire; jamais on ne voit mieux représentée une activité fondamentale de la vie des gouvernants. On pourrait aussi bien retenir des images de guerre, la naissance avortée de Tancrède, qui en restera nain, les étapes de l'onction impériale (d'abord l'onction des mains, puis des bras, la remise de l'épée, du sceptre, de l'anneau et enfin de la mitre), une mappemonde.

2 La présentation détaillée de l'installation des Staufen en Sicile est donnée en introduction par Th.K. Ensuite le même auteur se consacre à l'oeuvre et son auteur. La première fut réalisée entre 1195 et 1197 . Du second, on sait par lui-même qu'il était un clerc poète (son colophon dit: »Mon maître Pierre d'Eboli, fidèle serviteur de l'empereur, j'ai composé ce livre pour l'honneur d'Auguste«). Certes l'historien porte son attention sur les données événementielles et leur interprétation (il s'agit ici de légitimer la prise de pouvoir de Henri VI, comme la Tapisserie de Bayeux légitimait la conquête de Guillaume), mais il parait plus tentant d'admirer l'habileté du schéma iconographique, si rare à ce point de détail, si peu souvent appliqué à un texte historique. Le travail réalisé par les éditeurs a été important, et le traducteur et le commentateur ont droit en particulier à notre gratitude. Il serait bon qu'un tel ouvrage soit largement dans les instituts d'histoire du Moyen Age.

3 Avec la famille RÖSCH et leur livre sur Frédéric II, on entre en contact avec le fils tant espéré de l'union du souverain allemand et de l'héritière inattendue du royaume de Sicile. Voici un livre singulier. Passé le titre et le sommaire, on plonge immédiatement dans le sujet, sans introduction, ni remerciement, ni explication du partage des rôles entre le deux auteurs; une telle simplicité est rare. Et ce dans quoi on est plongé est d'abord un tableau discret et précis, fait de petites touches, c'est-à-dire de courts paragraphes faisant le tour d'une question ou la posant (comme ce sera tout au long de l'ouvrage), de l'Occident autour de 1200; ce tableau a pour but d'énoncer des faits, d'aborder des domaines qui évolueront ensuite. Un second chapitre se concentre sur »'enfant des Pouilles« ou »'enfant d'Apulie«. Une centaine de pages permettent de suivre la montée en puissance de Frédéric II, selon la chronologie, sans y coller vraiment. Les dates précises, la volonté de suivre jusqu'au détail l'histoire de ce prince ne sont pas les soucis ordinaires des auteurs. C'est ce qui donne à leur livre sa qualité particulière, à savoir une approche historique légère et réfléchie, faisant fi de détails chronologiques et de données pesantes. On sent toujours le désir primordial d'informer, d'expliquer et de commenter, non pas celui de bâtir une biographie. On remarque particulièrement bien cette tendance dans les premiers chapitres, où sont passés en revue les innovations, les inventions, les aspects géniaux et originaux de Frédéric: le droit, la construction des églises et des châteaux, la vie des palais, l'intérêt pour la science et la culture; on va au-delà avec l'examen des objets de luxe, des manteaux de couronnement, des couronnes et de bijoux. Au hasard des pages, on rencontre les enfants, les épouses, l'entourage; au hasard des pages on en sait plus sur 
l'Allemagne (présentation discrète des fils Henri (VII) et Conrad (IV), sur les villes, sur Rome et la papauté.

4 Le livre est doublement complété, en premier d'un chapitre sur la postérité de celui qui fut la stupor mundi et que l'on ne pouvait croire mort, en second de plusieurs dossiers iconographiques bien choisis et très réussis: manuscrits, châteaux, pièces, portraits. Les RÖSCH n'ont cherché à concurrencer personne; ils se sont fixés sur la Sicile et nous promènent paisiblement et avec goût et sensibilité à travers un règne particulier et un souverain fascinants; ils le font avec bonheur et nous ravissent.

5 Michel PARISSE 